\title{
An outburst of SS 433 observed on milliarcsecond scale
}

\author{
Valeriu Tudose ${ }^{* 1}$, Zsolt Paragi ${ }^{2,3}$, Rob Fender ${ }^{4}$, Mike Garrett ${ }^{1,5}$, James Miller-Jones ${ }^{6}$, \\ Sergei Trushkin ${ }^{7}$, Anthony Rushton ${ }^{8}$, Ralph Spencer ${ }^{9}$, George Heald ${ }^{1}$, Paolo \\ Soleri ${ }^{10}$ \\ ${ }^{1}$ Netherlands Institute for Radio Astronomy \\ ${ }^{2}$ Joint Institute for VLBI in Europe \\ ${ }^{3}$ MTA Research Group for Physical Geodesy and Geodynamics \\ ${ }^{4}$ University of Southampton \\ ${ }^{5}$ University of Leiden \\ ${ }^{6}$ Curtin Institute of Radio Astronomy \\ ${ }^{7}$ Special Astrophysical Observatory \\ ${ }^{8}$ European Southern Observatory \\ ${ }^{9}$ University of Manchester \\ ${ }^{10}$ University of Groningen \\ E-mail: tudosedastron.n]
}

\begin{abstract}
SS 433 is a high-mass X-ray binary system (XRB) and one of the most persistent sources of relativistic jets in the Milky Way. The object has been intensively studied in radio at arcsecond scales, however the high-resolution observations (i.e. VLBI) are relatively scarce. In 2008 November the system was in outburst. Using the e-VLBI capabilities of the European VLBI Network (EVN) we observed SS 433 for three epochs during the active phase. The data offered a detailed view of the system's behaviour in outburst at milliarcsecond scales. We used the "kinematic model" (which predicts the position along the jet of any knot ejected at some particular time in the past) to investigate the dynamic parameters of SS 433 and we examined the polarization properties of the ejected material. We report here the preliminary results.
\end{abstract}

10th European VLBI Network Symposium and EVN Users Meeting: VLBI and the new generation of radio arrays

September 20-24, 2010

Manchester UK

${ }^{*}$ Speaker. 


\section{SS 433}

SS 433 is an X-ray binary system (XRB) comprising a compact object, likely a black hole [10, 20], accreting matter from a companion star of relatively high mass [4]. The XRB is located at a distance of $5.5 \mathrm{kpc}$ [3] and has an orbital period of 13.1 days [5]. The object is embedded in a radio nebula, W50 [13, 6] and features a corkscrew-shaped double-sided radio jet on arcsecond scales (e.g. [22, 24, 16]). The radio jet is oriented close to the plane of the sky and is precessing with a period of 162.5 days (e.g. [1], 11]). Extended X-ray emission (i.e. jets) has been also observed [14, 15]. This emission is transient, oriented along the direction of the radio jets. The exact connection between the radio and X-ray emissions is however still elusive [16].

The radio jet has been extensively observed at arcsecond scales. Its derived geometrical parameters are consistent with those obtained at other wavelengths: $78.8^{\circ}$ inclination to the line of sight, $19.8^{\circ}$ opening angle of the precession cone, $0.26 c$ jet speed [24]. The arcsecond scale radio jet is linearly polarized. The fractional linear polarization ranges between $20-30 \%$, with the exception of a region within about 0.5 arcsecond from the core, where the radiation is depolarized [18, 25, 16]. Circular polarization has also been detected at a level below 1\% [9].

Observations at milliarcsecond scales are relatively scarce (e.g. [21, 19, 8, 27, 17, 2]). At these higher resolutions, the apparently continuous structure of the jet seen at arcsecond scales is resolved into individual, compact radio emitting blobs. Also notable is the presence of an "equatorial wind" extending from the core in a plane perpendicular to the jets, as observed projected on the sky [17].

\section{Observations}

We observed SS 433 at $5 \mathrm{GHz}$ with the European VLBI Network (EVN) in real-time e-VLBI mode on three occasions, 2008 November 6, 13, and 19, following an episode of major flaring activity in the system [26]. Standard calibration was applied using AIPS and imaging was carried out in Difmap. The resulting radio maps are presented in Fig. 1.

\section{The kinematic model}

The "kinematic model" of SS 433 is a geometric model for the precession (and nutation) of the jets in the system [1, 11]. Restricting the discussion to the radio wavelengths, basically it predicts the positions along the jet of various blobs of matter that have been ejected at particular moments in the past. The kinematic model has been tested extensively at arcsecond scales and on relatively fewer occasions at milliarcsecond scales. The parameters of the model are remarkably stable over periods of decades [ [7], although anomalies have been observed on a few occasions [19, 23, 12].

We applied the model to the three e-VLBI epochs using the parameter values listed in Table 1. We found that the precession of the jet alone can explain the general appearance of the system, however a significantly better match between the observations and the predictions of the model is obtained when the nutation of the jet is also taken into account (Fig. 1). Considering that the parameters in Table 1 have been determined using lower spatial resolution data at radio frequencies or data in the optical domain, the agreement between the model and the observations is notable. However, small discrepancies are present, as the kinematic model is an idealization. They could be 
Table 1: The precession and nodding parameters of the radio jet in SS 433 [24].

\begin{tabular}{lclc}
\hline \hline Precession parameter & Value & Nodding parameter & Value \\
\hline Inclination & $78^{\circ} .83$ & 5.8d Amplitude & 0.00382 \\
Cone angle & $19^{\circ} .85$ & 5.8d Period & $5.838 \mathrm{~d}$ \\
Position angle & $98^{\circ} .2$ & 5.8d Phase Reference & MJD 43588.0 \\
Phase reference & MJD 48615.5 & 6.3d Amplitude & 0.00655 \\
Period & $162.5 \mathrm{~d}$ & 6.3d Period & $6.290 \mathrm{~d}$ \\
Ejection velocity & $0.2602 \mathrm{c}$ & $6.3 \mathrm{~d}$ Phase Reference & MJD 43587.4 \\
\hline \hline
\end{tabular}

explained for instance by variations in the ejection velocity of the blobs of matter (or deceleration due to the interaction with the surrounding medium). Given the very limited time coverage of the observations (representing a small fraction of the precession period) it is not possible to use these data to refine the parameters listed in Table 1, but an attempt at the study of the behaviour of the radio emitting regions will be undertaken. This is even more relevant as the blobs imaged during the three epochs are situated within and near the so called "brightening zone" [27], a still not fully understood region where the flux densities of the individual blobs vary on timescales of days.

\section{Polarization at milliarcsecond scales}

Despite a number of studies (e.g. [25, 16]), the polarization properties of SS 433 at arcsecond scales are not fully understood. The observations are contradictory and there is still a debate whether the magnetic fields are preferentially oriented along the kinematic locus, or aligned with the local velocity vectors. High spatial resolution observations cannot discriminate between these two cases, but can offer information on the polarization properties close to the core of the system. These properties are virtually unknown at milliarcsecond scales. The only confident result seems to be that the region within about 0.5 arcsec of the core is relatively depolarized (more severely at lower frequencies), perhaps due to the presence of a strong wind [18, 25, 16].

We detected linear polarization of the order of a few percent $(<5 \%)$ in the core of the system in at least one of the three epochs (work in progress). This would be the first time when polarization is detected in SS 433 at such high spatial resolution (Fig. 2). What is to some extent puzzling is that the blobs separated from the core do not appear to be polarized (the $3 \sigma$ upper limit to the linearly polarized intensity is about $2 \mathrm{mJy} / \mathrm{beam})$. Assuming these components expand adiabatically and downstream in the jet interact with the surrounding medium, an ordered magnetic field should be generated that produces linear polarization of the radio emission. Alternatively, if instead of a fragmented, knotty structure, the jet of SS 433 is continuous, the bright knots are the result of shocks propagating downstream, a process that also generates polarized radio emission. Taking into account the low level of linear polarization present in the core and the fact that the individual knots are relatively less bright, one possible explanation for the lack of detection of polarization is an insufficient signal to noise ratio (assuming implicitly that the degree of linear polarization is of the same order as in the core). This will be further investigated by analyzing the polarization properties of the other two epochs and comparing them with the partial results presented here. 

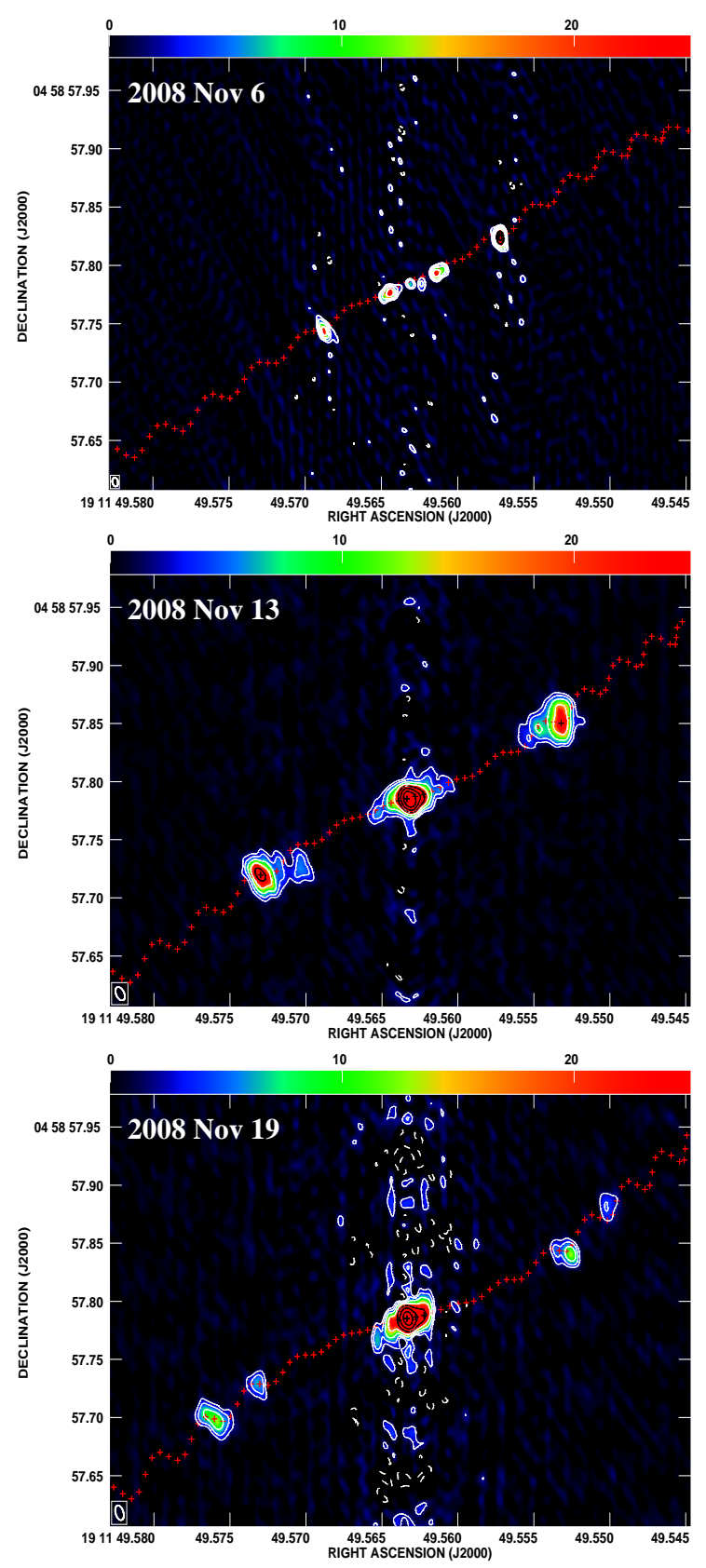

Figure 1: $5 \mathrm{GHz}$ e-EVN radio maps of SS 433. The contour levels are at $-1,1,2,4,8,16,32,64$ times the average $\mathrm{rms}$ noise of $2.0 \mathrm{mJy} / \mathrm{beam}$. The colour bars are expressed in $\mathrm{mJy} / \mathrm{beam}$. The images are at the same scale. The slightly different spatial resolutions are due to different radiotelescopes present in the arrays. Overlaid are the predictions of the kinematic model when both the precession and nutation of the jet are taken into account. The red marks correspond to the locus of hypothetical blobs of matter ejected at one day intervals. 


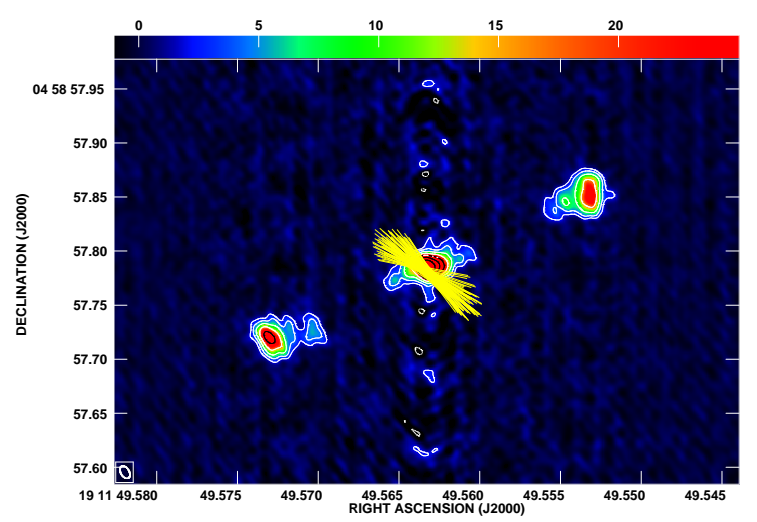

Figure 2: 5 GHZ e-EVN radio map of SS 433 from 2008 Nov 13 with the orientation of the electric vector position angles (EVPAs) superimposed. No galactic rotation measure correction and no PA calibration has been applied. The colour code bar is expressed in $\mathrm{mJy} / \mathrm{beam}$ and is associated to the total intensity map. For the polarization vectors 1 mas corresponds to $25 \mu \mathrm{Jy} / \mathrm{beam}$ linearly polarized intensity.

\section{Conclusions}

Preliminary analysis of 3 epochs of VLBI observations with the EVN of SS 433 during an outburst revealed the behaviour of the system at infrequently probed high spatial resolutions. We detected linear polarization in the system for the first time at milliarcsecond scales, at levels of the order of a few percent. The polarized emission is concentrated exclusively in the core, with none being detected in the knots tens of milliarcsecond away. At the moment, one likely explanation is a lack of enough signal to noise ratio for a confident detection outside the core. A more in depth analysis of the polarization properties is underway. We also tested the predictions of the kinematic model of the jet in SS 433 at milliarcsecond scales, where it has been relatively less well studied. We found that the model can explain the observations extremely well. However, discrepancies are present and the final analysis of the data might offer insights into the reasons behind them, possibly ruling out some of the explanations advanced based on lower spatial resolution data.

\section{Acknowledgments}

e-VLBI developments in Europe are supported by NEXPReS, an Integrated Infrastructure Initiative (I3), funded under the European Union Seventh Framework Programme (FP7/2007-2013) under grant agreement RI-261525. The European VLBI Network is a joint facility of European, Chinese, South African and other radio astronomy institutes funded by their national research councils.

\section{References}

[1] Abell G.O., Margon B., 1979, Nature, 279, 701

[2] Blundell K.M. et al., 2001, ApJ, 562, L79

[3] Blundell K.M., Bowler M.G., 2004, ApJ, 616, L159 
[4] Clark J.S., Barnes A.D., Charles P.A., 2007, MNRAS, 380, 263

[5] Crampton D., Hutchings J.B., 1981, ApJ, 251, 604

[6] Dubner G.M. et al., 1998, AJ, 116, 1842

[7] Eikenberry S.S. et al., 2001, ApJ, 561, 1027

[8] Fejes I., Schilizzi R.T., Vermeulen R.C., 1988, A\&A, 189, 124

[9] Fender R. et al., 2000, ApJ, 530, L29

[10] Hillwig T.C., Gies D.R., 2008, ApJ, 676, L37

[11] Hjellming R.M., Johnston K.J., 1981, ApJ, 246, L141

[12] Jowett F.H., Spencer R.E., 1995, in Proc. 27th YERAC, D.A. Green \& W. Steffen (eds.), p. 12

[13] Margon B., 1984, ARA\&A, 22, 507

[14] Marshall H.L., Canizares C.R., Schulz N.S., 2002, ApJ, 564, 941

[15] Migliari S., Fender R., Mendez M., 2002, Science, 297, 1673

[16] Miller-Jones J.C.A. et al., 2008, ApJ, 682, 1141

[17] Paragi Z. et al., 1999, A\&A, 348, 910

[18] Paragi Z. et al., 2004, in Proceedings of the 7th Symposium of the European VLBI Network, eds. R. Bachiller, F. Colomer, J.F. Desmurs and P. de Vicente, p. 221

[19] Romney J.D. et al., 1987, ApJ, 321, 822

[20] Seifina E., Titarchuk L., 2010, ApJ, 722, 586

[21] Schilizzi R.T. et al., 1981, Nature, 290, 318

[22] Spencer R.E. et al., 1984, MNRAS, 209, 869

[23] Spencer R.E., Waggett P., 1984, IAUS, 110, 297

[24] Stirling A.M. et al., 2002, MNRAS, 337, 657

[25] Stirling A.M. et al., 2004, MNRAS, 354, 1239

[26] Trushkin S.A., Nizhelskij N.N., 2008, ATel, 1819, 1

[27] Vermeulen R.C. et al., 1993, A\&A, 270, 177 